\title{
Suppression of convection around small magnetic concentrations
}

\author{
S. Morinaga ${ }^{1}$, T. Sakurai ${ }^{2}$, K. Ichimoto ${ }^{2}$, T. Yokoyama $^{3}$, M. Shimojo $^{4}$, and Y. Katsukawa ${ }^{2}$ \\ 1 Department of Astronomy, University of Tokyo, 7-3-1 Hongo, Bunkyo-ku, Tokyo 113-0033, Japan \\ e-mail: shuji.morinaga@nao.ac.jp \\ 2 National Astronomical Observatory of Japan, 2-21-1 Osawa, Mitaka, Tokyo 181-8588, Japan \\ 3 Department of Earth and Planetary Science, University of Tokyo, 7-3-1 Hongo, Bunkyo-ku, Tokyo 113-0033, Japan \\ 4 Nobeyama Radio Observatory, National Astronomical Observatory of Japan, Minamimaki, Minamisaku, Nagano 384-1305, Japan
}

Received 8 November 2007 / Accepted 16 January 2008

\section{ABSTRACT}

\begin{abstract}
Aims. It is well known that convective motions in the photosphere are suppressed by magnetic fields. However, it has been difficult to study the interaction between convection and small magnetic features, such as G-band bright points (GBPs) or pores with polarimetric measurements, because of the available spatial resolution $\left(\sim 1^{\prime \prime}\right)$. This situation is changed by the advent of the SpectroPolarimeter (SP) aboard the Hinode satellite, which has 0!'3 spatial resolution.

Methods. We analyzed the pore and its surrounding region in NOAA 10940 near the disk center. We obtained the field strength and filling factor through the Milne-Eddington inversion of the Stokes profiles. We also derived the line-of-sight velocity by the shift of the line core. Using these physical parameters, we investigated the physical conditions needed to suppress the convection.

Results. We found that the convection is suppressed, not by the strength of the magnetic field itself, but by high concentration of magnetic flux tubes. We also found that GBPs and pores are distinguished in terms of the filling factor $(f) ; f \simeq 0.6$ for GBPs and $f=0.8-0.9$ for pores.
\end{abstract}

Key words. Sun: photosphere - Sun: magnetic fields

\section{Introduction}

Pores differ from sunspots because they consist of an umbra without a penumbra. The typical size of pores is a few thousand kilometers, smaller than for sunspots. Although pores could be at a certain phase in the evolution of sunspots, they have not been studied more thoroughly than sunspots or small magnetic elements like G-band bright points (GBPs). With the spectro-polarimetric measurements using the Advanced Stokes Polarimeter (ASP; Elmore et al. 1992), Keppens \& Martinez Pillet (1996) studied sunspots and pores statistically. Leka \& Skumanich (1998) observed the time series of one event growing from a pore to a sunspot and presented their magnetic configurations and velocity structures. Skumanich (1999) investigated the evolution of pores and sunspots by considering the relation between their radii and their fluxes. Leka \& Steiner (2001) have found a ring structure of enhanced Stokes- $V$ area asymmetry around pores and show that it was caused by the downdraft located at the outer boundary of the pores.

Small pores, such as micro pores, sometimes have almost the same size as for granules. Therefore, high-resolution observations are required for studying the interaction between pores and granulation. However, because it takes time to obtain a wide field of view with spectro-polarimetric scans, temporally-changing atmospheric seeing prevents obtaining high resolution uniformly over the field of view. The $1^{\prime \prime}$ spatial resolution of ASP is comparable to the size of granules and makes it difficult to see these interactions clearly. Imaging observations are easier than spectroscopy to achieve high spatial resolution, but there is still much work to be done on these interactions. Keil et al. (1999) carried out multi-wavelength imaging spectroscopy using a birefringent filter and found that the downward flow velocity increases with depth in the photosphere. Hirzberger (2003) detected the downdraft at the outer boundary of pores by FabryPerot imaging spectroscopy, which is in good agreement with the previous work of Leka \& Steiner (2001). Because the SpectroPolarimeter (SP) aboard the Hinode satellite (Kosugi et al. 2007; Tsuneta et al. 2007) is free of atmospheric seeing, SP achieves a stable resolution of 0.3 , i.e. as high as an imaging observation. Morinaga et al. (2007) find that the center-to-limb variations of the Stokes- $V$ area asymmetry can be interpreted by the existence of the downdraft around the pores.

In this paper, we study the interaction between convective motions and the magnetic field around pores using SP and investigate the necessary physical condition for the suppression of convection. In Sect. 2, we explain the data to analyze. The result of Stokes inversion and the line-of-sight (LOS) velocity is shown in Sect. 3. Discussions follow in Sect. 4, and we conclude in Sect. 5.

\section{Observation}

The observation was carried out on 31 January 2007 looking at the active region NOAA 10940, which was located almost at the disk-center $(\mu=0.96)$. Simultaneous spectro-polarimetric measurements with SP and imaging with the Filtergraph (FG) were performed. The SP measures the Stokes profiles $(I(\lambda), Q(\lambda)$, $U(\lambda)$, and $V(\lambda)$ ) of the Zeeman-sensitive Fe I $6301.51 \AA$ and $6302.50 \AA$ line pair. The FG observation was at a 7-min cadence, taking G-band images. In this paper, we focus on the area in and around pores. Figure 1 shows the whole region that is analyzed. 


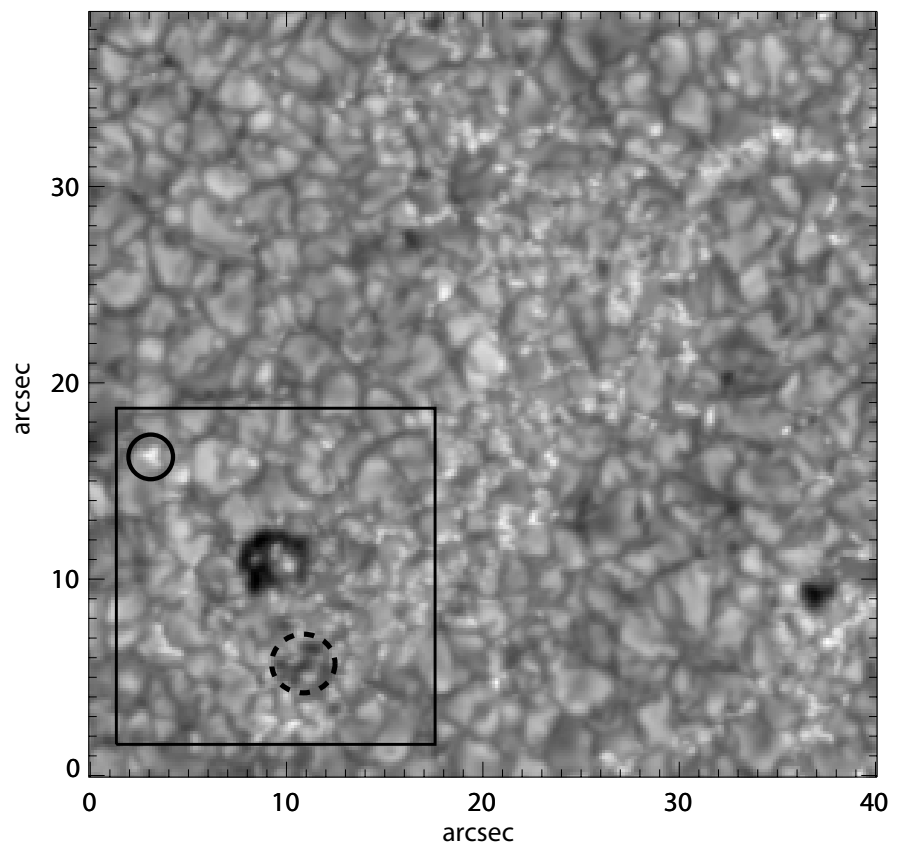

Fig. 1. The FG G-band image of the NOAA region 10940. The FOV is $40{ }^{\prime} 1 \times 39$.' 0 . The rectangle marks the region shown in Fig. 2. The solid and dashed circles indicate a G-band bright point and a small pore, respectively.

This region consisted of a plage region and was uni-polar. The size of the field of view was 40.' $1 \times 39$.' $^{\prime}$, and it took $22 \mathrm{~min}$ to be scanned by SP. The region marked by the square in Fig. 1 is enlarged in Fig. 2 showing the image of the magnetic field strength, the distributions of the filling factor (see the definition given below), and the LOS velocity around the pore. We used the FG G-band data, which were taken at the moment closest to the time when the center of the pore was scanned with SP. The G-band data were co-aligned to the continuum image of SP.

The magnetic field parameters were obtained through the Milne-Eddington (ME) inversion method (Skumanich \& Lites 1987). The ME inversion is a simple least-square method that reproduces the most reasonable Stokes profile via the model atmosphere in which the source function is a linear function of the optical depth, and other physical parameters do not change with height. The inversion gives, among other things, the magnetic field strength $B$ and the magnetic filling factor $f$. The latter is defined as the fraction of area in a pixel covered with the magnetic field, whereas the rest of the area in the pixel is field-free. In our study, we used the ME inversion code MEKSY developed by Yokoyama et al. (2007). The reliability of the filling factor obtained by the ME inversion applied to the SP data was confirmed by Orozco Suarez et al. (2007). The line-of-sight velocity $v_{\text {LOS }}$ was obtained from the shift in the Stokes $I$ line core of Fe I $6301.51 \AA$ with respect to the line center position of the mean quiet Sun intensity profile.

\section{Results}

In Fig. 2, we can see the convective pattern in the righthandside panel (LOS velocity) even in the region with strong magnetic field strength; e.g., the area above the dashed circle where the granular blueshift and intergranular redshift are seen while the field strength is more than $1 \mathrm{kG}$ and the filling factor is less than 0.3. However, the convective motions are suppressed within the region of high filling factor. Figure 3 is a scatter plot between the continuum intensity of SP and the filling factor, created from the whole area displayed in Fig. 1. We divide the data points into three groups according to the continuum intensity. The continuum intensity of group I is lower than 0.9 , and the group I data points include the pore area. The intensity of group II is between 0.9 and 1.1, and that of group III is more than 1.1 , respectively. Group I has higher mean filling factor $\left(\left\langle f_{\mathrm{I}}\right\rangle \simeq 0.55\right)$ than the other two groups. The highest values of the filling factor in group I correspond to the pore. Group II corresponds to the area of typical granules. The group II data points with lower continuum intensity are seen in the intergranular lanes, while those with higher intensity are seen near the center of granules in the continuum image. The filling factor of this group shows a wide range of distribution between 0 and 1 with the mean $\left\langle f_{\text {II }}\right\rangle \simeq 0.25$. Group III has the lowest mean filling factor among the three groups, $\left\langle f_{\text {III }}\right\rangle \simeq 0.12$. The group III data points are found at the brightest parts of granules in the continuum image.

Figure 4 shows a scatter plot between the line-of-sight velocity and the filling factor. The dispersion in the LOS velocity is smallest for group I $\left(\sigma_{\mathrm{I}}=0.10 \mathrm{~km} \mathrm{~s}^{-1}\right)$, and its mean LOS velocity shows redshift $\left(\left\langle v_{\mathrm{I}}\right\rangle=0.11 \mathrm{~km} \mathrm{~s}^{-1}\right)$. The group II data points show large dispersion in the LOS velocity $\left(\sigma_{\mathrm{II}}=0.13 \mathrm{~km} \mathrm{~s}^{-1}\right)$, and they are slightly redshifted $\left(\left\langle v_{\mathrm{II}}\right\rangle=0.05 \mathrm{~km} \mathrm{~s}^{-1}\right)$. Both group I and group II data points show redshift most clearly for $0.3<f<0.6$. Group III consists of data points around zero LOS velocity and a branch of data points directed toward blueshift. The latter is a manifestation of upward-moving hot plasma at the center of the granules. The dispersion in the LOS velocity is largest among the three groups $\left(\sigma_{\text {III }}=0.14 \mathrm{~km} \mathrm{~s}^{-1}\right)$, and the mean LOS velocity is blueshifted $\left(\left\langle v_{\text {III }}\right\rangle=-0.13 \mathrm{~km} \mathrm{~s}^{-1}\right)$. When the three groups are combined, the dispersion in the LOS velocity decreases with the increase in the filling factor for $0.2<f<0.8$.

Figure 5 is a scatter plot between the LOS velocity and the magnetic field strength. Although we can see a concentration of data points around $1300 \mathrm{G}$, the dispersion in the LOS velocity shows similar values regardless of the magnetic field strength if the field is more than $500 \mathrm{G}$.

Figure 6 is a scatter plot between the LOS velocity and the mean magnetic field strength. The mean magnetic field strength is the product of the filling factor and the magnetic field strength. If $A$ is the area that corresponds to one pixel on the solar surface, and $S$ is the cross section of the flux tube within a pixel, the mean magnetic field strength is given by

$f B=\frac{S B}{A}=\frac{\Phi}{A}$

where $\Phi$ is the total magnetic flux in one pixel. The dispersion in the LOS velocity clearly decreases with the increase in the mean magnetic field strength or the total magnetic flux within a pixel. If we only look at data points with low values of mean field strength (say, $f B<300 \mathrm{G}$ ), the group I data points are on average redshifted and the group III data points are blueshifted.

The blueshift-side branch of group III, seen in Fig. 4 and corresponding to the center of granules, is buried in the left bottom skirt in Fig. 6, and its mean field strength is weak $(f B<300 \mathrm{G})$. The group III data points with a high filling factor and low LOS velocity in Fig. 4 are embedded in group II data points and have the same property as the brightest data points of group II. Indeed, if we choose a higher intensity threshold for group III, these data points are merged into group II, whereas the blueshift-side branch remains.

The part of group I data points in Fig. 4 that have a small filling factor is located in the right bottom skirt in Fig. 6 and 

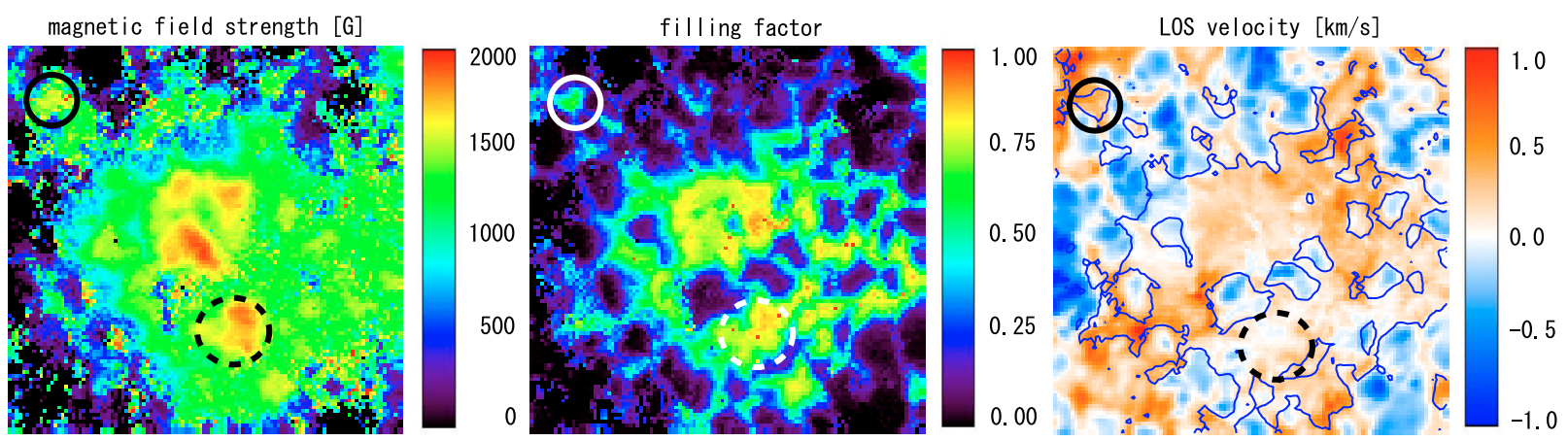

Fig. 2. The magnetic field strength map (left), the distribution of the filling factor $f$ (middle), and the LOS velocity map (right) of a portion of the analyzed region centered on the region around a large pore. The solid and dashed circles indicate a G-band bright point and a small pore, respectively (same as in Fig. 1). Contours in the right-hand-side panel represent $f=0.3$.

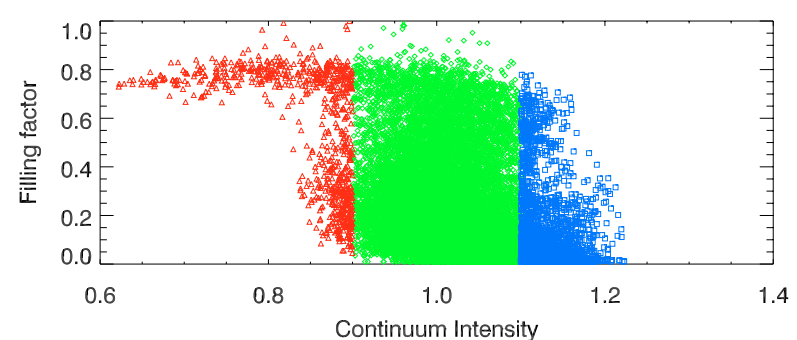

Fig. 3. Scatter plot between the normalized continuum intensity of the $I$ profile $\left(I_{\mathrm{c}}\right)$ and the magnetic filling factor. The continuum intensity, $I_{\mathrm{c}}$, is normalized to the averaged continuum intensity of the quiet region at the disk center. The data points are divided into three groups in terms of their brightness. Group I (triangles), II (diamonds), and III (squares) are defined as $I_{\mathrm{c}}<0.9,0.9 \leq I_{\mathrm{c}} \leq 1.1$, and $I_{\mathrm{c}}>1.1$, respectively.

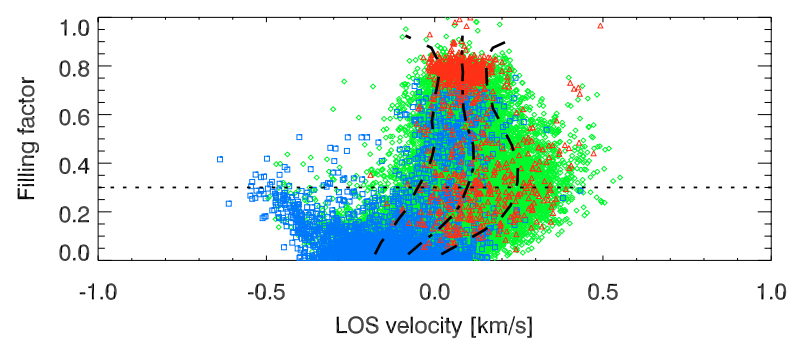

Fig. 4. Scatter plot between the LOS velocity and the filling factor. The positive (negative) line-of-sight velocity means the redshift (blueshift). The meaning of the symbols and lines is the same as in Fig. 3. The dashdotted line shows the averaged LOS velocity and dashed lines show its dispersion calculated for bins of 0.05 width in the filling factor, taking all groups into account. The dotted horizontal line represents $f=0.3$, and the LOS velocity shows a smaller dispersion above this line.

its mean field strength is weak. They are basically the same as the darkest part of group II. These data points are located in the intergranular lanes, and show redshift and weak field strength in Fig. 5. Because of the low filling factor and low field strength, the convection is not suppressed and a high redshift is seen.

By comparing the image of the G-band intensity with the map of the filling factor, we can recognize that GBPs and pores are located in regions where the filling factor is higher than 0.5 (see Fig. 2). Therefore, the high filling factor is presumably responsible for the suppression of convection. We discuss this point in the next section. Moreover, the G-band feature marked

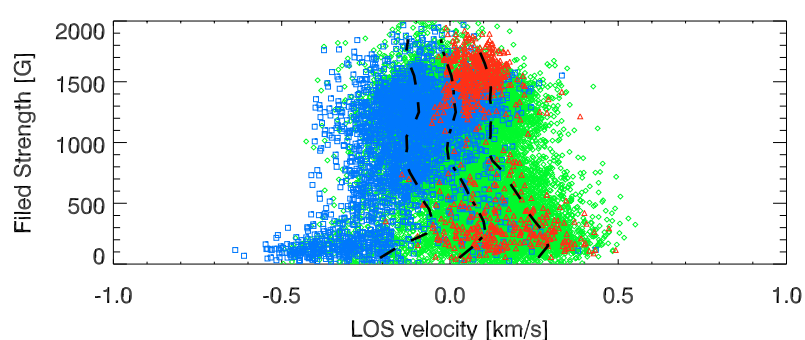

Fig. 5. Scatter plot between the LOS velocity and the magnetic field strength $(B)$. The meaning of the symbols and lines is the same as in Fig. 3. The dash-dotted line shows the averaged LOS velocity and dashed lines show its dispersion calculated for bins of $100 \mathrm{G}$ width in the field strength, taking all groups into account.

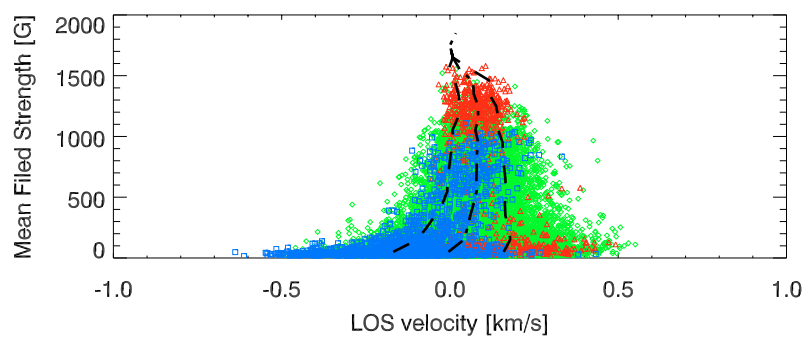

Fig. 6. Scatter plot between the LOS velocity and the mean magnetic field strength (or magnetic flux, $f B$ ). The meaning of the symbols and lines is the same as in Fig. 3. The thick lines are the same as in Fig. 5.

with the solid circle almost has the same size as the pore marked with the dashed circle. The magnetic field strengths in both features are around $1600 \mathrm{G}$, without any significant difference between them. Although they have the same property concerning the size and the magnetic field strength, their appearance differs; one is observed as a pore and the other is observed as a bright G-band structure (a GBP or an aggregate of GBPs). This result suggests that the difference in whether the structure appears as a pore or a bright G-band structure is caused by the difference in the filling factor. The filling factor for a structure to be bright in the G-band is around 0.6, while pores are formed for a filling factor of $0.8-0.9$.

There have been several studies of the size and the total flux within small magnetic elements, such as GBPs, magnetic knots (Beckers \& Schröter 1968), and pores. Spruit \& Zwaan (1981) and Knoelker \& Schuessler (1988) have predicted that 
there could be a "smooth transition" from bright magnetic features, e.g., GBPs (Berger et al. 1995; Wiehr et al. 2004) to pores or proto-pores (Hirzberger 2003). The penumbra generally develops if the radius of the magnetic structure exceeds 1750 km (Bray \& Loughhead 1964; Leka \& Skumanich 1998). Theoretically, a stable existence of dark magnetic features requires the magnetic flux exceeding $10^{19} \mathrm{Mx}$ (Meyer et al. 1977). This flux translates into the size of $800 \mathrm{~km}$ assuming a field strength of $2000 \mathrm{G}$ for pores. However, Wiehr et al. (2004) discovered that the diameter of inter-granular bright points (i.e. the magnetic components of the GBPs) is $160 \mathrm{~km}$ and its upper limit is $300 \mathrm{~km}$. These results contradict the "smooth transition" of the magnetic features. However, part of the discrepancy might come from the difficulty in identifying small dark features as pores, because a portion of the intergranular lanes is as dark as in pores. In our case, however, we have magnetic data so that we are able to distinguish real dark magnetic features (pores) from non-magnetic dark features. As seen in Fig. 2, the small pores or proto-pores there have the size of about $500 \mathrm{~km}$ and flux of about $3.2 \times 10^{18} \mathrm{Mx}$. These small dark features may fill the gap between the classical pores and the bright points.

\section{Discussion}

We have found that the pores are characterized by high field strength and a large filling factor. If the field strength is universally close to a kilogauss (in our example $1300 \mathrm{G}$ ), then the pores are said to be formed in regions of high filling factor. We have also observed that the convection (represented by the dispersion in the LOS velocity) is suppressed within regions of high filling factor. What counts in suppressing the convection is not the field strength itself but the filling factor, because there is little variation in the dispersion of the LOS velocity with the magnetic field strength. In contrast, convective motions are suppressed more if the mean magnetic field strength (or the total flux) within a pixel is greater.

The convective motion may set in if the kinetic energy of a certain volume exceeds the magnetic energy in the same volume. Here we assume that the convective motion may try to overturn the magnetized and non-magnetized volumes altogether. This means that we assume $v_{\mathrm{NM}}=v_{\mathrm{MAG}}=v_{\mathrm{LOS}}$, where $v_{\mathrm{NM}}$ and $v_{\mathrm{MAG}}$ is the LOS velocity of non-magnetized and magnetized components, respectively. A threshold value for the filling factor can be derived by considering the energy balance between the magnetic field $\left(f B^{2} / 8 \pi\right)$ and the convective motions $\left(\rho v^{2} / 2\right)$. We obtain

$f_{\text {crit }}=\frac{4 \pi \rho v^{2}}{B^{2}}$.

Assuming the mass density $\rho=2.51 \times 10^{-7}\left[\mathrm{~g} \mathrm{~cm}^{-3}\right]$ at the surface (OPAL) and the convective velocity $v=2.0 \times 10^{5}\left[\mathrm{~cm} \mathrm{~s}^{-1}\right]$ in the quiet region, and the magnetic field strength of the magnetic elements to be $1300[\mathrm{G}]$, we obtain $f_{\text {crit }} \simeq 0.07$. (On the other hand, the so-called equi-partition field of $350 \mathrm{G}$ corresponds to $f=1$ for the same values of $\rho$ and $v$.) However, the kinetic energy driving the granular convection should be represented by some kind of average density $\rho$ over the depth of the granules. For this average and if we take $\rho=1.07 \times 10^{-6}\left[\mathrm{~g} \mathrm{~cm}^{-3}\right]$ (OPAL), then we obtain $f_{\text {crit }}=0.32$. Observationally $f_{\text {crit }} \simeq 0.3$, because in Fig. 2 we can clearly see the suppression of convection and weak redshift in the region $f>0.3$. The convective blue shift and granular patterns are seen in the region $f<0.3$.
We also noticed that the pores are surrounded by a magnetic region with a high (about 0.5 ) filling factor, while the surroundings of the GBPs are less magnetic. The convection is therefore expected to be suppressed in the region surrounding the pores. If the size of such a region exceeds the photon mean-free path, the radiation from the hot wall of the flux tube (Spruit \& Zwaan 1981; Solanki 1986) cannot heat the interior of the tube and will help cool the pores. The downdraft observed inside the GBPs suggests that the convection is not entirely suppressed in the GBPs.

\section{Conclusions}

We analyzed the active region NOAA 10940 with emphasis on the pores and the surrounding granules, using the SP and FG data of Hinode. We obtained the magnetic parameters through the Milne-Eddington inversion of the Stokes profiles, and the line-of-sight velocity from the shift of the intensity profile. The dispersion in the LOS velocity tells us that the suppression of the convection is related not to the field strength but to the mean magnetic field strength over the pixel or the total magnetic flux within it. The key parameter controlling the convective motions is the magnetic filling factor, or equivalently, the mean magnetic field strength. The convection is suppressed if $f$ exceeds the threshold of about 0.3 . The difference between the pores and the GBPs can also be explained by the filling factor; $f \simeq 0.6$ for GBPs, $f \simeq 0.8-0.9$ for pores. And their environment is probably related to the difference in the appearance of GBPs and pores.

Acknowledgements. Hinode is a Japanese mission developed and launched by ISAS/JAXA, with NAOJ as domestic partner and NASA and STFC (UK) as international partners. It is operated by these agencies in co-operation with ESA and NSC (Norway). This work was partly carried out at the NAOJ Hinode Science Center, which is supported by the Grant-in-Aid for Creative Scientific Research, the Basic Study of Space Weather Prediction from MEXT, Japan (Head Investigator: K. Shibata), generous donations from Sun Microsystems, and NAOJ internal funding. The authors thank Dr. J. Jurcak for useful discussion.

\section{References}

Beckers, J. M., \& Schröter, E. H. 1968, Sol. Phys., 4, 142 Berger, T. E., Schrijver, C. J., Shine, R. A., et al. 1995, ApJ, 454, 531 Bray, R. J., \& Loughhead, R. E. 1964, Sunspots, The International Astrophysics Series (London: Chapman Hall)

Elmore, D. F., Lites, B. W., Tomczyk, S., et al. 1992, in Polarization analysis and measurement, ed. D. H. Goldstein, \& R. A. Chipman, Proc. SPIE, 1746, 22 Hirzberger, J. 2003, A\&A, 405, 331

Keil, S. L., Balasubramaniam, K. S., Smaldone, L. A., \& Reger, B. 1999, ApJ, 510,422

Keppens, R., \& Martinez Pillet, V. 1996, A\&A, 316, 229

Knoelker, M., \& Schuessler, M. 1988, A\&A, 202, 275

Kosugi, T., Matsuzaki, K., Sakao, T., et al. 2007, Sol. Phys., 243, 3

Leka, K. D., \& Skumanich, A. 1998, ApJ, 507, 454

Leka, K. D., \& Steiner, O. 2001, ApJ, 552, 354

Meyer, F., Schmidt, H. U., \& Weiss, N. O. 1977, MNRAS, 179, 741

Morinaga, S., Nagata, S., Ichimoto, K., et al. 2007, PASJ, 59, S613

Orozco Suarez, D., Bellot Rubio, L. R., del Toro Iniesta, J. C., et al. 2007, PASJ, in press

Skumanich, A. 1999, ApJ, 512, 975

Skumanich, A., \& Lites, B. W. 1987, ApJ, 322, 473

Solanki, S. K. 1986, A\&A, 168, 311

Spruit, H. C., \& Zwaan, C. 1981, Sol. Phys., 70, 207

Tsuneta, S., Suematsu, Y., Ichimoto, K., et al. 2007, Sol. Phys., submitted

Wiehr, E., Bovelet, B., \& Hirzberger, J. 2004, A\&A, 422, L63

Yokoyama, T., Katsukawa, Y., \& Shimojo, M. 2007, in preparation 